Running Head: UNDERREPORTING VIOLENCE AGAINST PARAMEDICS

\title{
The Role of Organizational Culture in Normalizing Paramedic Exposure to Violence
}

\author{
Justin Mausz $^{1,2,3}$, Mandy Johnston ${ }^{2}$, and Elizabeth Donnelly ${ }^{4}$
}

Keywords: Emergency Medical Services, Paramedic, Violence, Operational Stress Injury, Mental Health

\section{Author Note}

Justin Mausz (D) https://orcid.org/0000-0001-6955-5387

Elizabeth Donnelly (D) https://orcid.org/0000-0001-9942-3203

We have no conflicts of interest to disclose.

Correspondence concerning this article should be addressed to: Justin Mausz, Peel Regional Paramedic Services, Fernforest Reporting Station: 1600 Bovaird. Dr. E. Brampton, ON, L6V 4R5. justin.mausz@peelregion.ca

\footnotetext{
${ }^{1}$ Peel Regional Paramedic Services, Regional Municipality of Peel, Brampton, Ontario; ${ }^{2}$ The McNally Project for Paramedicine Research, Toronto, Ontario; ${ }^{3}$ McMaster University, Department of Health Research Methods, Evidence, and Impact, Hamilton, Ontario; ${ }^{4}$ The University of Windsor, School of Social Work, Windsor, Ontario, Canada
} 


\begin{abstract}
Purpose

Violence against paramedics is a complex - but underreported - problem. Extant research suggests organizational culture may play a role in sustaining cultural norms that downplay the significance and limit reporting. Our objective was to qualitatively explore paramedics' experience with violence, with particular emphasis on understanding how organizational culture contributes to under-reporting.
\end{abstract}

\title{
Approach
}

We surveyed paramedics from a single, large, urban service in Ontario, Canada, asking participants to describe their experiences with violence, including whether - and why or why not - the incidents were reported. Within a constructivist epistemology, we used inductive thematic analysis with successive rounds of coding to identify and then define features of organizational culture that limit reporting.

\section{Findings}

A total of 196 (33\% of eligible) paramedics completed the survey. Fully $98 \%$ of participants disclosed having experienced some form of violence; however only a minority (40\%) reported the incidents to management, or the police $(21 \%)$. We defined a framework within which a lack of support from management, and consequences for offenders, implicitly positions the ability of 
paramedics to "brush off" violent encounters as an expected professional competency.

Disclosing emotional or psychological distress in response to violent encounters invited questions as to whether the individual is personally suited to paramedic work.

\section{Originality}

While the extant research has indicated that underreporting is a problem, our findings shed light on why - a critical first step in addressing what has been described as a serious public health problem.

Keywords: Paramedic, Violence, Qualitative Research, Operational Stress Injury, Mental Health 
UNDERREPORTING VIOLENCE AGAINST PARAMEDICS

\section{Introduction}

Violence against Emergency Medical Services (EMS) personnel is a complex and growing problem (Murray et al., 2020; Pourshaikhian et al., 2016). A number of cross-sectional survey studies have suggested that a concerning majority of EMS workers have experienced some form of violence within the past calendar year (Alharthy et al., 2017; Bigham et al., 2014; Furin et al., 2015; Maguire, Browne, et al., 2018; Maguire \& O'Neill, 2017), including verbal abuse, threats, and physical and sexual assault, including assaults with weapons. This creates the risk of potentially significant physical (Maguire, Browne, et al., 2018; Maguire et al., 2005; Maguire et al., 2002) and psychological (Kim et al., 2019; Knor et al., 2020; Wongtongkam, 2017) harms. As a result, violence against EMS personnel has been described as a serious public health problem (Maguire \& O'Neill, 2017), but despite the growing recognition of the potential for harm, violent incidents often go unreported (Bigham et al., 2014; Maguire, Browne, et al., 2018; Maguire, O'Meara, et al., 2018). For example, in a cross-sectional survey of more than 1,500 paramedics in two provinces in Canada, Bigham and colleagues found that despite $75 \%$ of surveyed paramedics having experienced a violent encounter within the past 12 months, less than $40 \%$ reported the incident to a supervisor or police, and fewer than $20 \%$ documented the incident at all (Bigham et al., 2014). In their qualitative analysis of survey comments, participants described exposure to violence as "part of the job" (p. 492), despite explaining that being subjected to violence at work negatively affected their job performance, mental health, and family life (Bigham et al., 2014). These sentiments echo a growing body of research that suggests that features of the organizational culture within the profession may contribute to underreporting (Maguire, O'Meara, et al., 2018; Murray et al., 2020). This significantly limits the 
ability of EMS organizations to enhance the safety of their personnel through improved policies, procedures, training, or changes to legislation. Indeed, a recent systematic review proposed a research agenda that identified, among other things, the need for reliable epidemiological surveillance data (Murray et al., 2020). Any effort, however, at improving surveillance requires the buy-in of the EMS personnel. In that respect, better understanding the reasons for underreporting is an important first step in working to address the problem of violence against EMS providers. Here, the extant research has been sparse in explaining the specific mechanisms by which exposure to workplace violence becomes normalized as 'part of the job'. It is concerning that such a serious risk to both physical and psychological safety can be dismissed so casually, and the sentiment hints at an underlying process within the professional culture that enables the belief to persist. Our objective, therefore, was to qualitatively explore reasons for underreporting, with a particular emphasis on understanding the potential role of organizational culture within a single paramedic service in Ontario, Canada.

\section{Methods}

Overview

As part of a broader effort within the paramedic service to mitigate the risk of workplace violence, we distributed a web-based survey to our personnel with the goals of exploring paramedic experiences with workplace violence and reasons for underreporting. The survey consisted of a combination of multiple choice and open-ended free text questions, was distributed via workplace email, and was made available for a 30-day period. Situated within a 
constructivist epistemology (Creswell, 2013), we adopted a qualitative descriptive approach (Sandelowski, 2010), using inductive thematic analysis (Vaismoradi et al., 2013) to derive an explanatory framework to explore how features of organizational culture contribute to underreporting incidents of violence.

The [institution blinded for peer review] provided ethics review and approval (REB \#19-207) for this work as a secondary use of data originally intended for quality improvement purposes. Consequently, the requirement for informed participant consent (for research purposes) was waived, although all paramedics within the service were assured that participation in the survey was voluntary and that their responses were anonymous.

\section{Setting}

Our study was situated in a single paramedic service in Ontario, Canada. [Service name blinded for peer review] is a publicly funded, sole provider of land ambulance services to the [blinded for peer review], encompassing a mixed suburban and rural geography of $1,200 \mathrm{~km}^{2}$ with a population of 1.5 million residents. At the time of the survey, [the service] employed more than 600 primary and advanced care paramedics (P/ACPs) and responded to an average of 130,000 emergency calls per year, making the service the second largest by caseload and staffing within the province of Ontario.

\section{Survey}


The survey was created using the web-based platform SurveyMonkey and was circulated via workplace email to all employees maintaining certification to practice as a paramedic $(n=632)$. The survey was available for the 30 -day period between February $14^{\text {th }}$ to March $14^{\text {th }}, 2019$ with emails sent at 2-week intervals encouraging participation. Survey questions consisted of demographic information including years of experience and gender, as well as a combination of forced-response (i.e., yes/no, multiple choice) and free-text questions designed to explore paramedic experiences with exposure to violence. In describing different types of workplace violence, we drew on the definitions used by Bigham et al. in their 2014 study. Survey items were generated through consensus by a working group composed of both front-line paramedics and management, with a draft of the survey sent to a regional information analysis team for external consultation and feedback. The final version of the survey (Table 1) was approved by the service's senior administration prior to distribution.

[Table 1 about here]

\section{Data Analysis}

Quantitative data were analyzed using summary and descriptive statistics to report on the proportion of survey participants reporting exposure to violence, the types of violence encountered, and the actions taken (i.e., reporting). Qualitative data in the form of free-text narrative comments were assembled in Microsoft Word and imported into NVivo for Mac Version 11 (QSR International) for analysis. Our analysis followed the principles of inductive thematic analysis (Braun \& Clarke, 2014; Vaismoradi et al., 2013), borrowing the coding 
methods of grounded theory (Charmaz, 2014b). In thematic analysis, researchers identify descriptive themes while remaining analytically 'close' to the data in a manner that allows for interpretive flexibility but avoids deep inference or high levels of abstraction (Vaismoradi et al., 2013). Grounded theory coding methods, meanwhile, emphasize explicating social processes within the data to construct explanatory frameworks or theories (Charmaz, 2014b). This began with open coding, assigning short labels to segments of text using gerunds to identify social actions (Charmaz, 2014b). Subsequent rounds of focused coding deleted, collapsed, and expanded the original open codes as appropriate, organizing the codes by conceptual similarity (Charmaz, 2014a). A final round of coding clarified the relationships between conceptual categories and organized the codes into a coherent explanatory framework. Our analysis was situated within a constructivist epistemology (Creswell, 2013), in which the subjectivity of participant experiences, and the role of the researcher in the construction of knowledge is embraced, rather than bracketed out (Carter \& Little, 2007). Data coding was carried out jointly by two investigators, who, themselves, are practicing paramedics within the service. We used invivo annotations and memos (Charmaz, 2014c) to document analytical decisions during the coding process and enhance reflexivity, and met with the broader project team regularly to discuss in-progress work. These meetings were especially helpful in ensuring that the framework we developed was described presented in a way that resonate with end-users in developing policy interventions to address violence against paramedics.

\section{Results}

"It's part of the job; you learn to deal with it, or the job becomes unbearable." 
A total of 196 paramedics completed the survey out of a pool of 588 eligible (i.e., not on longterm leave) participants for a response rate of $33.3 \%$. Fifty percent $(\mathrm{N}=98)$ of the participants were women, $48 \%$ (94) were men, with 2 participants providing another, non-binary gender. Most participants (46\%) had more 10 years of experience, with the remainder evenly split between the $0-5$ year (27\%) and 6-10 year (26\%) experience categories. With respect to exposure to violence during their careers, 191 participants (97.5\%) reported having experienced verbal abuse, $168(86.1 \%)$ intimidation, $156(80 \%)$ physical assault, $120(61.5 \%)$ sexual harassment, and $27(13.8 \%)$ sexual assault. Only a minority of participants indicated having reported the incident to service management $(40 \%)$, or to the police $(21.3 \%)$. The free text responses yielded 63 single-spaced pages of comments for a total of 30,602 words for analysis.

[Table 2 about here]

Through successive rounds of coding, we defined a framework (Figure 1) within which chronic and widespread exposure to violence is perceived as being unpreventable and without consequence for offenders. Within this construction, the ability of paramedics to simply "brush off" violent encounters become an expected professional competency. We describe the components of our framework below.

Note to Readers 
The survey questions touched on difficult topics, and the participants occasionally used strong language, which - for the purposes of presenting their experiences faithfully, we have not redacted. The excerpts we present may be triggering to readers who have personal or professional experience with violence.

[Figure 1 about here]

\section{Widespread and Chronic Violence}

The experiences of abuse that the participants described were shocking. Our participants disclosed having been subjected to name calling and racial slurs, threatening behaviour, physical attacks (sometimes with weapons), sexual harassment, and sexual assault. The overarching commonality was in the diversity of the abuse described, and the frequency with which it was encountered; nearly every participant reported experiencing various types of abuse, often more than once. This litany of abuse ranged from relatively benign incidents - such as being yelled at or called offensive names - to more serious incidents, including being threatened with death or rape, or being physically or sexually assaulted.

"At the extreme end, I have been threatened with knives twice (i.e., the weapons were presented and held against me)."

"I've entered a residence and found someone pointing a gun at me."

"I have been chased out of a house while having objects (including knives) being thrown at me." 
"As a woman, having men think it is their right to comment on a female's look, or put their hand up a medic's thigh, or 'accidentally' grab a breast, all regardless of the paramedic's verbal warnings."

Collectively, the participants explained that exposure to violence in the course of their duties is both widespread and chronic, often commenting that they have experienced "too many (incidents) to count". This, in large part, contributed to the perception that being subjected to violence is simply unpreventable.

\section{Perceived as Unpreventable}

The participants expressed that the nature of paramedic work lends itself to being exposed to violence. Because paramedics respond to emergencies in the community where the environment is difficult to control, emotions run high, and the patients (or others at the scene) are often affected by alcohol or drugs, the perception was that the risk of violence cannot be eliminated or even meaningfully reduced.

"I think a lot of the violence is just inherent in the job and cannot be curbed. When we are dealing with patients with mental health problems, alcohol and drug impairments, or medical issues (i.e., diabetes, seizures), they either have no control over their behaviour or don't care to control their behaviour."

Desirable as it might be, the participants expressed that neither the paramedics themselves, or the service more broadly, can prevent people from acting violently: "We cannot control the actions of others." As a result, the participants explained that violence "will always occur and will remain a 'staple' of our job." 
UNDERREPORTING VIOLENCE AGAINST PARAMEDICS

\section{A Lack of Consequences for Offenders}

Compounding the view of violence as an unpreventable feature of paramedic work was the added perception that there are often no consequences for people who commit acts of violence against paramedics. Because much of the violence that the participants described was perpetrated by people under the influence of alcohol or drugs, or whose judgement and cognition were otherwise impaired, the police were often reluctant to pursue charges: "If the patient is drunk, medical (postictal, hypoglycemic), on drugs, or ANY medical reason, (the police) can't do anything about it." Even when the paramedics described being injured as a result of an assault, they were told that the prospect of criminal prosecution was unlikely:

"I finished the call and was seen in the ER (emergency room) by a doctor, and I was off (work) with a wrist injury for 3 months. My supervisor was notified as well as the police, and I was told it was up to me if I wanted to press charges but that it may not go anywhere."

The result was twofold: first, it compounded the problem of underreporting, because the perception was that there is "no point in reporting violence; nothing will be done", and second, it left the affected paramedics with a profound sense of injustice.

"It makes me feel worthless. It shows society that they can get away with this behaviour towards paramedics because there are no consequences." 
Collectively, chronic and widespread violence, its perception as being unpreventable, combined with a lack of consequences for offenders, normalized exposure to violence as an expected feature of paramedic work.

"We deal with so many drunk and drugged folks who act out towards us. We can't (sedate) them all, nor can we throw them all in (jail) - you can't lock people up for being assholes - and we can't just up and walk away from the call either."

The perceived inevitability of violence shifted the focus away from the perpetrators and onto the paramedics. The result was that the participants explained they were expected to tolerate violence, often in service of professionalism.

"Someone can assault you and you have to remember that you are in uniform and representing (the service) before you can respond."

"It made me feel vulnerable and that it's okay for me to be treated that way because the patient and their family are in a state of emergency and I need to tolerate the situation."

"I feel like I am unable to stand up for myself during these situations, as 'professionalism' seems to take priority over whatever the paramedic has experienced and how it has affected them."

This despite sometimes experiencing significant physical or psychological harm as a result of a violent incident. For example, our participants described a wide range of difficult emotions after being the victims of a violent encounter, including guilt, shame, and self-doubt as a result of wondering whether they had done something to provoke the incident or done enough to prevent it. 
"I have a hard time processing the intense emotions I feel - fear, anger, frustration, inadequacy, guilt - did I provoke this?"

Meanwhile, other participants reported having developed post-traumatic stress disorder (PTSD) after a violent encounter, with some unable to return to work.

"I have also developed PTSD and am once again off work ... I still hope to return to full duties at some point, but we will have to see how that goes."

When the survey participants disclosed having been affected by a violent incident to coworkers or supervisors, they were often met with an unsympathetic response. The normalization of violence as an expected feature of paramedic work implicitly positioned the ability of paramedics to simply "brush off" and "move on from" violent encounters as an expected professional competency, the inability to do so inviting questions about their suitability for paramedic work. For example, one participant described a situation where, after having been assaulted, she was found crying in the back of her ambulance by two supervisors:

"The two (supervisors) on that night found me hiding in the back of the ambulance crying. In a nutshell, I was told I'm probably not cut out for this job and should start looking for a new career. 'Look at yourself; you're a mess.",

There was, however, an awareness that this culture needs to change, and that even though violence may not necessarily be preventable, the paramedics should not be expected to tolerate it as just being "part of the job".

"If I go to a restaurant and yell at the staff, I get kicked out, and rightfully so. But, if I call 911 and attempt to physically or verbally assault the paramedics, then it is just "part of what they signed up for". It is complete bullshit." 


\section{Discussion}

Our goal was to better understand why paramedics are reluctant to report incidents of workplace violence and the role that organizational culture within the service may have in contributing to underreporting. While a number of previous investigations have identified that exposure to workplace violence is often considered 'part of the job' (Bigham et al., 2014; Murray et al., 2020), what has been lacking is a more nuanced understanding of why these sentiments persist and what the potential consequences are.

What we found was that the experience of violence as being widespread and chronic led to the perception of it being unpreventable. Because so much of the violence that our participants described was perpetrated by people whose judgement was impaired, the belief was that most of the violent incidents simply cannot be avoided. Complicating this perception was the experience that there are rarely consequences for those who commit acts of violence. While criminal prosecution may not have been appropriate in many, or even the majority of cases that our participants described, the perceived lack of consequences for offenders created a profound sense of injustice. The net result of chronic, 'unpreventable', and 'unprosecutable' violence was that the exposure was normalized, and - by extension - the ability for the participants to 'brush off' individual incidents was implicitly positioned as an expected professional competency. Those who could not were often met with questions about their suitability for paramedic work. What this suggests is that any effort to address the problem and mitigate the risk of violence must consider the broader context and organizational culture within which it occurs. 
One potential first step in addressing features of organizational culture within EMS that limit reporting is to engage the providers themselves in the development of reporting processes. In their 2014 report, Bigham and colleagues identified the importance of securing the buy-in of paramedics as a necessary precondition in the development of reporting processes and specifically recommended that EMS personnel be involved during the design stage (Bigham et al., 2014). In our own service, we have recently launched a novel, prospective, point-of-event reporting mechanism and associated 'back end' procedures for following up on incidents of violence. The development and implementation have been described elsewhere [Reference blinded for peer review], but in brief, the process involved a lengthy stakeholder consultation and pilot testing phase within which the paramedic experience was centered. When a report is filed, a supervisor contacts the paramedics to provide post-incident follow-up. This may include, for example, contacting the police, facilitating medical care, completing an accident-injury report, and referring the paramedics to appropriate mental health resources. Where the risk of recurrence is high, an address hazard flag can be generated to alert subsequent paramedic crews attending the scene of the potential for violence. This follow-up process is part of the development of comprehensive strategy within the service to mitigate the risk of harm from exposure to violence and includes increased collaboration with the local police service, crisis prevention and intervention training, 'zero tolerance for violence' stickers within all ambulances, and a public awareness campaign to draw attention to the issue.

Better understanding the prevalence, types, experiences, and potential long-term consequences of exposure to violence is an important priority for both researchers and EMS leadership alike. A growing body of research suggests that EMS personnel experience high rates of 'Operational 
Stress Injuries' (OSIs) (Berger et al., 2012; Wagner et al., 2020), including post-traumatic stress disorder (PTSD), depression, anxiety (R. N. Carleton et al., 2018), and chronic pain (Carleton et al., 2017), that - combined with exposure to trauma (Carleton et al., 2019) - conspire to place EMS personnel at an increased risk of suicide (R. Nicholas Carleton et al., 2018). Existing research suggests that the risk of work-related PTSD is the result of a combination of both acute and chronic workplace stressors, particularly in the setting of alcohol use, collectively accounting for $\sim 40 \%$ of the variability in self-reported PTSD symptoms (Donnelly, 2012). While exposure to violence has been examined within the context of defining subsets of emergency calls with the potential for psychological distress ('critical incidents') (Carleton et al., 2019; Donnelly \& Bennett, 2014), the specific contribution to the risk of OSI remains unclear.

Understanding and mitigating the long-term impact of violence requires research approaches that prospectively and longitudinally evaluate exposure to violence and its potential psychological sequelae. It is possible, in fact, that the very act of engaging paramedics in the development of reporting and follow-up processes may not only ameliorate barriers to reporting, but also reduce the risk of any attendant psychological harm by validating and supporting the short-term emotional responses that can result from potentially traumatic events (Bryant et al., 2000). Not all trauma leads to PTSD (Carleton et al., 2019; Kyron et al., 2021), and what separates 'exposure' from 'outcome' likely has more to do with the organizational response following a 'critical incident' rather than the event itself - a hypothesis which is increasingly supported in the paramedic mental health literature (Halpern et al., 2009a, 2009b; Halpern et al., 2011, 2014). Moreover, addressing organizational barriers to reporting may have the added benefit of mitigating other chronic workplace stressors within the organization. 


\section{Limitations}

Our findings should be interpreted within the context of certain limitations. First, we acknowledge that our response rate of $33 \%$ is below what is conventionally held to be an acceptable threshold for generalizability for survey research. We note, however, that our goal in this analysis was not to estimate prevalence, for which a higher response rate or more robust sampling strategy would be required, but rather to qualitatively explore the experience of the survey participants themselves. In that respect, our analytical approach was positioned to offer us unique insights in a previously unexplored area of inquiry. We also acknowledge, though, that the analysis of unstructured survey comments may not yield the fulsome insights that more indepth data gathering methods (such as interviewing or field observations) can produce (LaDonna et al., 2018). All of this is to say that our findings are contextually bound and invite further study in other settings with other methods to enhance our collective understanding of the issues.

\section{Conclusion}

In our study, we identified a number of features of organizational culture that conspire to limit the reporting of incidents of violence against EMS. When violence is widespread and chronic, perceived as unpreventable and without consequence for offenders, the exposure can become normalized as an accepted feature of EMS work - and, by extension, tolerance of violence an implicit professional competency. Shifting this perception requires the buy-in of EMS personnel, the benefits of which will not only enhance our understanding of the epidemiology of this 
important health issue, but also potentially mitigate its long-term psychological impacts. Taken together, efforts to enhance reporting represents an important priority for both research and policy.

\section{Acknowledgements}

[Removed for blinding purposes.]

\section{Declaration of Conflicting Interests}

The authors declare that there is no conflict of interest. 
UNDERREPORTING VIOLENCE AGAINST PARAMEDICS

\section{References}

Alharthy, N., Mutairi, M. A., Alsahli, A., Alshehri, A., Almatrafi, A., Mahah, A., Khalid Alswailem, A., Phiulip, W., \& Qureshi, S. (2017). Workplace violence among emergency medical services workers in Riyadh, Saudi Arabia. Journal of Hospital Administration, 6(3). https://doi.org/10.5430/jha.v6n3p26

Berger, W., Coutinho, E. S., Figueira, I., Marques-Portella, C., Luz, M. P., Neylan, T. C., Marmar, C. R., \& Mendlowicz, M. V. (2012). Rescuers at risk: a systematic review and meta-regression analysis of the worldwide current prevalence and correlates of PTSD in rescue workers. Soc Psychiatry Psychiatr Epidemiol, 47(6), 1001-1011. https://doi.org/10.1007/s00127-011-0408-2

Bigham, B., Jensen, J. L., Tavares, W., Drennan, I., Saleem, H., Dainty, K. N., \& Munro, G. (2014). Paramedic self-reported exposure to violence in the emergency medical services (EMS) workplace: A mixed-methods cross sectional survey. Prehosp Emerg Care, 18, 489-494.

Braun, V., \& Clarke, V. (2014). What can "thematic analysis" offer health and wellbeing researchers? Int J Qual Stud Health Well-being, 9, 26152. https://doi.org/10.3402/qhw.v9.26152

Bryant, R. A., Moulds, M. L., \& Guthrie, R. M. (2000). Acute stress disorder scale: A self-report measure of acute stress disorder. Psychol Assess, 12(1), 61-68. https://doi.org/10.1037//I040-3590.12.1.61

Carleton, R. N., Afifi, T. O., Taillieu, T., Turner, S., Krakauer, R., Anderson, G. S., MacPhee, R. S., Ricciardelli, R., Cramm, H. A., Groll, D., \& McCreary, D. R. (2019). Exposures to 
UNDERREPORTING VIOLENCE AGAINST PARAMEDICS

potentially traumatic events among public safety personnel in Canada. Canadian Journal of Behavioural Science / Revue canadienne des sciences du comportement, 51(1), 37-52. https://doi.org/10.1037/cbs0000115

Carleton, R. N., Afifi, T. O., Turner, S., Taillieu, T., Duranceau, S., LeBouthillier, D. M., Sareen, J., Ricciardelli, R., MacPhee, R., Groll, D., Hozempa, K., Brunet, A., Weekes, J. R., Griffiths, C. T., Abrams, K. J., Jones, N. A., Beshai, S., Cramm, H. A., Dobson, K. S., Hatcher, S., Keane, T. M., Stweart, S. H., \& Asmundson, J. G. (2018). Mental disorder symptoms among public safety personnel in Canada. Can J Psychiatry, 63(1), 54-64. https://doi.org/10.1177/0706743717723825

Carleton, R. N., Afifi, T. O., Turner, S., Taillieu, T., El-Gabalawy, R., Sareen, J., \& Asmundson, G. J. G. (2017). Chronic pain among public safety personnel in Canada. Canadian Journal of Pain, 1(1), 237-246. https://doi.org/10.1080/24740527.2017.1410431

Carleton, R. N., Afifi, T. O., Turner, S., Taillieu, T., LeBouthillier, D. M., Duranceau, S., Sareen, J., Ricciardelli, R., MacPhee, R. S., Groll, D., Hozempa, K., Brunet, A., Weekes, J. R., Griffiths, C. T., Abrams, K. J., Jones, N. A., Beshai, S., Cramm, H. A., Dobson, K. S., Hatcher, S., Keane, T. M., Stewart, S. H., \& Asmundson, G. J. G. (2018). Suicidal ideation, plans, and attempts among public safety personnel in Canada. Canadian Psychology/Psychologie canadienne, 59(3), 220-231. https://doi.org/10.1037/cap0000136

Carter, S. M., \& Little, M. (2007). Justifying knowledge, justifying method, taking action: epistemologies, methodologies, and methods in qualitative research. Qual Health Res, 17(10), 1316-1328.

Charmaz, K. (2014a). Focused coding and beyond. In J. Seaman (Ed.), Constructing Grounded Theory (2nd ed., pp. 138-161). Sage Publications. 
UNDERREPORTING VIOLENCE AGAINST PARAMEDICS

Charmaz, K. (2014b). The logic of grounded theory coding practices and initial coding. In J. Seaman (Ed.), Constructing Grounded Theory (2nd ed., pp. 109-137). Sage Publications.

Charmaz, K. (2014c). Memo-writing. In J. Seaman (Ed.), Constructing Grounded Theory (2nd ed., pp. 162-191). Sage Publications.

Creswell, J. W. (2013). Philosophical assumptions and interpretive frameworks. In L. Habib (Ed.), Qualitative inquiry \& research design: Choosing among five approaches (3 ed., pp. 15-41). SAGE Publications.

Donnelly, E. (2012). Work-related stress and posttraumatic stress in emergency medical services. Prehosp Emerg Care, 16(1), 76-85. https://doi.org/10.3109/10903127.2011.621044

Donnelly, E. A., \& Bennett, M. (2014). Development of a critical incident stress inventory for the emergency medical services. Traumatology: An International Journal, 20(1), 1-8. https://doi.org/10.1177/1534765613496646

Furin, M., Eliseo, L. J., Langlois, B., Fernandez, W. G., Mitchell, P., \& Dyer, K. S. (2015). Selfreported provider safety in an urban emergency medical system. West J Emerg Med, 16(3), 459-464. https://doi.org/10.5811/westjem.2015.2.24124

Halpern, J., Gurevich, M., Schwartz, B., \& Brazeau, P. (2009a). Interventions for critical incident stress in emergency medical services: a qualitative study. Stress and Health, 25(2), 139-149. https://doi.org/10.1002/smi.1230

Halpern, J., Gurevich, M., Schwartz, B., \& Brazeau, P. (2009b). What makes an incident critical for ambulance workers? Emotional outcomes and implications for intervention. Work \& Stress, 23(2), 173-189. https://doi.org/10.1080/02678370903057317 
UNDERREPORTING VIOLENCE AGAINST PARAMEDICS

Halpern, J., Maunder, R. G., Schwartz, B., \& Gurevich, M. (2011). Identifying risk of emotional sequelae after critical incidents. Emerg Med J, 28(1), 51-56. https://doi.org/10.1136/emj.2009.082982

Halpern, J., Maunder, R. G., Schwartz, B., \& Gurevich, M. (2014). Downtime after critical incidents in emergency medical technicians/paramedics. Biomed Res Int, 2014, 483140. https://doi.org/10.1155/2014/483140

Kim, J. H., Lee, N., Kim, J. Y., Kim, S. J., Okechukwu, C., \& Kim, S. S. (2019). Organizational response to workplace violence, and its association with depressive symptoms: A nationwide survey of 1966 Korean EMS providers. $j$ Occup Health, 61(1), 101-109. https://doi.org/10.1002/1348-9585.12025

Knor, J., Pekara, J., Seblova, J., Peran, D., Cmorej, P., \& Nemcova, J. (2020). Qualitative Research of Violent Incidents Toward Young Paramedics in the Czech Republic. West J Emerg Med, 21(2), 463-468. https://doi.org/10.5811/westjem.2019.10.43919

Kyron, M. J., Rees, C. S., Lawrence, D., Carleton, R. N., \& McEvoy, P. M. (2021). Prospective risk and protective factors for psychopathology and wellbeing in civilian emergency services personnel: a systematic review. J Affect Disord, 281, 517-532. https://doi.org/10.1016/j.jad.2020.12.021

LaDonna, K. A., Taylor, T., \& Lingard, L. (2018). Why Open-Ended Survey Questions Are Unlikely to Support Rigorous Qualitative Insights. Acad Med, 93(3), 347-349. https://doi.org/10.1097/ACM.0000000000002088

Maguire, B. J., Browne, M., O'Neill, B. J., Dealy, M. T., Clare, D., \& O'Meara, P. (2018). International Survey of Violence Against EMS Personnel: Physical Violence Report. Prehosp Disaster Med, 33(5), 526-531. https://doi.org/10.1017/S1049023X18000870 
Maguire, B. J., Hunting, K. L., Guidotti, T. L., \& Smith, G. S. (2005). Occupational injuries among emergency medical services personnel. Prehosp Emerg Care, 9(4), 405-411. https://doi.org/10.1080/10903120500255065

Maguire, B. J., Hunting, K. L., Smith, G. S., \& Levick, N. R. (2002). Occupational fatalities in emergency medical services: a hidden crisis. Ann Emerg Med, 40(6), 625-632. https://doi.org/10.1067/mem.2002.128681

Maguire, B. J., O'Meara, P., O'Neill, B. J., \& Brightwell, R. (2018). Violence against emergency medical services personnel: A systematic review of the literature. Am J Ind Med, 61(2), 167-180. https://doi.org/10.1002/ajim.22797

Maguire, B. J., \& O'Neill, B. J. (2017). Emergency Medical Service Personnel's Risk From Violence While Serving the Community. Am J Public Health, 107(11), 1770-1775. https://doi.org/10.2105/AJPH.2017.303989

Murray, R. M., Davis, A. L., Shepler, L. J., Moore-Merrell, L., Troup, W. J., Allen, J. A., \& Taylor, J. A. (2020). A Systematic Review of Workplace Violence Against Emergency Medical Services Responders. New Solut, 29(4), 487-503. https://doi.org/10.1177/1048291119893388

Pourshaikhian, M., Abolghasem Gorji, H., Aryankhesal, A., Khorasani-Zavareh, D., \& Barati, A. (2016). A Systematic Literature Review: Workplace Violence Against Emergency Medical Services Personnel. Arch Trauma Res, 5(1), e28734. https://doi.org/10.5812/atr.28734

Sandelowski, M. (2010). What's in a name? Qualitative description revisited. Research in Nursing \& Health, 33, 77-84. 
Vaismoradi, M., Turunen, H., \& Bondas, T. (2013). Content analysis and thematic analysis:

Implications for conducting a qualitative descriptive study. Nursing and Health Sciences, $15,398-405$.

Wagner, S. L., White, N., Regehr, C., White, M., Alden, L. E., Buys, N., Carey, M. G., Corneil, W., Fyfe, T., Matthews, L. R., Randall, C., Krutop, E., \& Fraess-Phillips, A. (2020). Ambulance personnel: Systematic review of mental health symptoms. Traumatology, 26(4), 370-387. https://doi.org/10.1037/trm0000251

Wongtongkam, N. (2017). An exploration of violence against paramedics, burnout and posttraumatic symptoms in two Australian ambulance services. International Journal of Emergency Services, 6(2). 
